Sebastian Starystach*

\title{
Zur Funktion informeller Kommunikation in Gerichtsverfahren am Beispiel des deutschen Strafverfahrens
}

https://doi.org/10.1515/sosys-2017-0004

Zusammenfassung: Die qualitative Untersuchung arbeitet die Funktion informeller Kommunikation in Gerichtsverfahren am Beispiel des deutschen Strafverfahrens heraus. Informelle Kommunikation ermöglicht in diesem Kontext Angeklagte auf die Übernahme einer geskripteten Verfahrensrolle einzustellen. Durch Lernen im sozialen System der juristischen Profession werden Quellen möglichen Protests gegen das Urteil neutralisiert. Diese Arbeit an der personalen Umwelt zur Bewältigung von Inklusionsproblemen findet maßgeblich in der Experten-LaienBeziehung zwischen Mandant und Verteidiger statt. Dreh- und Angelpunkt der Arbeit an der personalen Umwelt ist ein dem Konditionalprogramm des Rechts gehorchender Fall, der kollegial auf der Hinterbühne mit dem Ziel einer zeitökonomisch effizienten Aufführung der mündlichen Verhandlung hergestellt wurde. Nach Lösung der Inklusionsprobleme können die Mechanismen von Legitimation durch Verfahren, die die Institutionalisierung von Protest nach enttäuschter Erwartung verunmöglichen, auf der Vorderbühne der mündlichen Verhandlung greifen.

Abstract: The study investigates the function of informal communication in court proceedings using the example of German criminal proceedings. Informal communication prepares defendants to assume a scripted procedural role. By learning in the social system of the legal profession, sources of possible protests against the decision are neutralized. This work on the personal environment serves to cope with inclusion problems and takes place mainly in the expert lay relationship between client and defender. The scripting is based on a case that obeys the conditional program of the law and which was produced collegially on the backstage with the aim of its time-economically efficient performance in the oral proceedings. Once the inclusion problems are solved, the mechanisms of legitimization through procedure that make it impossible to institutionalize protest after disappointed expectation can be effective on the frontstage of the oral proceedings.

*Korrespondenzautor: Dr. Sebastian Starystach, Ruprecht-Karls-Universität Heidelberg, Max-Weber-Institut für Soziologie, Bergheimer Straße 58, 69115 Heidelberg, Deutschland, email: sebastian.starystach@soziologie.uni-heidelberg.de 


\section{Einleitung}

Laut Ergebnissen aktueller Bevölkerungsbefragungen genießen in Deutschland die Justiz und Verwaltung im Vergleich zu anderen demokratischen Institutionen umfangreiches Vertrauen. ${ }^{1}$ Auf der Ebene des medialen Diskurses gerät jedoch das Handeln von an Gerichtsverfahren beteiligten Juristen regelmäßig in den Fokus der Kritik. Vor allem in öffentlichkeitswirksamen Strafverfahren steht wiederholt der Verdacht im Raum, dass Rechtspraktiker aus zeitökonomischen, politischen, persönlichen oder sonstigen Gründen durch informelle Machenschaften rechtsstaatliche Verfahrensstandards unterlaufen würden. Auch der juristische Diskurs beschäftigt sich seit mehr als dreißig Jahren mit dem Phänomen der informellen Verfahrensabsprache (aktuell vgl. Altenhain/Dietmeier/May 2013; Brand/Petermann 2010; Fischer 2010; Hettinger 2011; Nahrwold 2014; Pfister 2013; Schreiber-Klein 2015; Theile 2012). Die hier prominent diskutierte Frage nach der Bedrohung rechtsstaatlicher Prinzipien durch Absprachen hat bereits das Bundesverfassungsgericht beschäftigt (Bundesverfassungsgericht 2013). ${ }^{2}$

Aus soziologischer Perspektive erscheinen hingegen der Rechtstaat und seine Verfahren auf professionelle nichtöffentliche Interpretationsleistungen und informelle Aushandlungsprozesse angewiesen, um sich in ihrer Formalität gegenüber Umweltanforderungen behaupten zu können (Holzinger 2015). „Informell“ bezeichnet dabei alle „Operationen, die sich außerhalb der Logik formaler Prinzipien des Rechtsstaats bewegen“ (Holzinger 2015, 7), d.h. nicht kodifiziert sind und auf intersubjektiv geteilten Realitätsdefinitionen beruhen. Mit der informellen Anwendung des Konditionalprogramms des Rechts in Gerichtsverfahren durch damit betreute Professionsmitglieder sind folglich zentrale Fragen der „kontinuierlichen Institutionalisierung von Recht“ (Stegmaier 2009, 391f.) berührt, wobei die verfahrensspezifische „Formalitäts-Informalitäts-Spanne“ (Elias 1989, 41) als ein zentrales Systemcharakteristikum verstanden werden muss.

Dabei entsteht ein systematisches Dilemma in Bezug auf die Legitimation von Verfahrensergebnissen: Rechtliche Verfahren genießen gesellschaftliche

\footnotetext{
1 So vertrauten im Frühjahr 2018 72\% der Bundesbürger der Justiz (26\% vertrauten ihr nicht), $71 \%$ der öffentlichen Verwaltung (25\% vertrauten ihr nicht). Hingegen vertrauten lediglich $59 \%$ dem Parlament (37 \% vertrauten ihm nicht) und nur $36 \%$ den politischen Parteien (60\% vertrauten ihnen nicht) (Europäische Kommission 2018, T39, T40, T43, T46).

2 Das Bundesverfassungsgericht hatte zu entscheiden, ob die Vorgaben des in 2009 eingeführten § 257c StPO durch informelle Absprachen systematisch unterlaufen würden und damit zusammenhängend die grundgesetzlich verankerten Rechtsstaatsprinzipien der „Selbstbelastungsfreiheit“, des „Schuldprinzips“, die mit diesem verbundene „Pflicht zur Erforschung der materiellen Wahrheit“ sowie das „Recht auf ein faires Verfahren“ bedroht würden.
} 
Anerkennung, und wenn ihre formalen Bestimmungen wie der Strafprozessordnung (StPO) eingehalten werden, realisieren sie sich als öffentliche, transparente und faire Geschehnisse legitimer Etikettierung. ${ }^{3}$ Gleichzeitig ist aber in einem gewissen - jedoch empirisch unbekannten Umfang - informelle professionelle Kommunikation notwendig, um formelle rechtstaatliche Strukturen wie das Strafverfahren dauerhaft gegenüber Umweltanforderungen funktionsfähig zu halten. Informelle Kommunikation bedroht allerdings die Legitimität von Verfahrensergebnissen, da nicht mehr öffentlich nachvollziehbar ist, auf welchen Grundlagen die Entscheidung ruht.

Der Beitrag von Luhmanns „Legitimation durch Verfahren“ (1983) ${ }^{4} \mathrm{zu}$ diesem Problemkomplex kann nicht überschätzt werden. Luhmann versteht Legitimität als „eine generalisierte Bereitschaft, inhaltlich noch unbestimmte Entscheidungen innerhalb gewisser Toleranzgrenzen hinzunehmen“ (1983, 28). Verfahren sichern dadurch Legitimität, dass diejenigen, die von Entscheidungen betroffen sind, öffentlich an ihrer Herstellung beteiligt werden. Es ist eine systematische soziologische Reflexion auf die Implikationen formalrechtlicher Verfahren im Hinblick auf die Akzeptanz von Entscheidungen. Bei aller Kritik (Machura 1993) ermöglicht sie, als Heuristik die latente Funktion von formalen Verfahren als Prozess legitimer Etikettierung im Sinne einer „frontstage“ systematisch ins Verhältnis zu setzen mit informeller Kommunikation auf der „backstage“ bzw. „back region“ (Goffman 1990, $111 \mathrm{ff}$.).

Vor diesem theoretischen Hintergrund fragt die folgende Untersuchung danach, (a) welche Funktion informeller Kommunikation bei Herstellung von Verfahrensergebnissen zukommt und (b) in welchem Verhältnis sie zur Legitimation von Verfahrensergebnissen steht. Dies wird mit Hilfe eines qualitativen Forschungsdesigns am Beispiel des deutschen Strafverfahrens herausgearbeitet, da hier die Anwendung verfahrensrechtlicher Maßstäbe und informelle Kommunikation umfangreich in einem Spannungsverhältnis zueinander stehen. ${ }^{5}$

Zur Beantwortung der Fragestellung werden theoretische Grundlagen des Mechanismus von Legitimation durch Verfahren mit dem Fokus auf Gerichtsverfahren dargelegt (Abschnitt 2.). Darauf aufbauend wird unter Rückbezug auf professionssoziologische Ansätze eine Heuristik für die empirische Unter-

3 Siehe zu „Fairness“ auch den Beitrag von Machura (2017) in diesem Heft.

4 Der Erstauflage von 1969 folgte 1975 eine zweite, um ein neues Vorwort erweiterte Auflage (beide im Luchterhand-Verlag). Nach einer dritten, unveränderten Auflage 1978 erscheinen alle weiteren Ausgaben seit 1983 im Suhrkamp-Verlag. Im Folgenden wird das Buch nach der Suhrkamp-Ausgabe von 1983 zitiert, die seiten- und textidentisch mit der dritten Auflage von 1978 ist. 5 Ausgeklammert werden in diesem genuin soziologischen Zugriff die mit dem „Handel mit Gerechtigkeit“ (Schumann 1977) verbundenen normativen Fragen. 
suchung herausgearbeitet (Abschnitt 3.). An die Darstellung des Forschungsstands (Abschnitt 4.), der Datengrundlage und des methodischen Vorgehens (Abschnitt 5.) anschließend wird die Funktion informeller Kommunikation im Kontext des Strafverfahrens herausgearbeitet (Abschnitt 6.). Die Arbeit schließt mit einer Reflexion der empirisch vorfindbaren Formalitäts-Informalitäts-Spanne und ihren Implikationen für die Legitimation von Verfahrensergebnissen im Strafverfahren (Abschnitt 7.).

\section{Legitimation durch Gerichtsverfahren}

Anders als zunächst anzunehmen, besteht die legitimierende Funktion von Verfahren nicht darin, den von einer Entscheidung Betroffenen von der Richtigkeit dieser zu überzeugen, sondern möglichen Protest gegen die Entscheidung zu neutralisieren. ${ }^{6}$ Legitimation durch Verfahren ist „Lernen im sozialen System“ (119) und das Ergebnis ist, dass die Entscheidung als verbindlich akzeptiert wird, „ohne daß es auf die innere Bereitschaft noch ankäme“ (117). Dieser Lernprozess wird durch die ko-produktive Erzeugung einer Verfahrensgeschichte ermöglicht, wobei die von der Entscheidung Betroffenen zentral an der Herstellung des Ergebnisses durch die Übernahme von Verfahrensrollen mitwirken (45).

Motiviert $\mathrm{zu}$ dieser öffentlichen Ableistung ,unbezahlter zeremonieller Arbeit“ (117) werden die Beteiligten durch die Ungewissheit über den Ausgang des Verfahrens, die im Falle des Gerichtsverfahrens über die Unabhängigkeit des Richters institutionalisiert ist (96ff.). Nur durch diese Ungewissheit ist gesichert, dass das jeweilige Rollenhandeln der Beteiligten auch die Möglichkeit hat, die Verfahrensgeschichte und damit den Verfahrensausgang $\mathrm{zu}$ beeinflussen sowie ggf. für sich zu bestimmen. Damit ist Ungewissheit des Verfahrensausgangs „die treibende Kraft des Verfahrens, der eigentlich legitimierende Faktor“ (116).

Durch die Übernahme der Verfahrensrollen wird Komplexität reduziert, da sich die Teilnehmer freiwillig und gleichberechtigt in Referenz zum Sachverhalt festlegen. Das Verfahren baut über die Verfahrensrollen systematisch einen Konsistenzzwang auf, der als „Trichter“ (115) fungiert und mögliches Rollenhandeln zunehmend einschränkt. Jedes eingespeiste Rollenhandeln (z. B. Aussage, Antrag, etc.), d.h. Information, entfaltet im Verfahren eine Bindewirkung, da nachfolgende Information an bisheriger in ihrer Konsistenz bemessen wird (z. B. Vorhaltung einer widersprüchlichen Aussage). Auf diese Weise wird der verhandelte Sachverhalt gemeinsam auf eine Entscheidung hin strukturiert. Dies kann

6 Siehe auch den Beitrag von Jost (2017) in diesem Heft. 
dann mit Entscheidungsprozessen - z. B. des Richters - synchronisiert werden, die zur Umwelt des Verfahrenssystems gehören (3).

Die gemeinsame Schaffung einer Verfahrensgeschichte reduziert aber nicht nur fallbezogene Komplexität; zudem wird von den Beteiligten durch formalrechtlich kodifizierte Partizipation das Verfahren als Mechanismus der Komplexitätsreduktion (implizit) anerkannt. Damit wird die reduzierte Komplexität intersubjektiv übertragbar (30), da im Falle des Protests darauf verwiesen werden kann, dass der von der Entscheidung Betroffene gleichberechtigt und freiwillig an ihrer Herstellung beteiligt war. In der Folge kann sein Protest „nicht zur Institution werden“ (120), da bei Aufrechterhaltung des Protests der von der Entscheidung Betroffene als deviant etikettiert und in der Rolle eines Abweichenden stabilisiert wird (118). Diese Möglichkeit der Externalisierung sozialer Kontrolle wird verfahrensintern dadurch gesichert, dass der Etikettierungsprozess öffentlich ist und damit eine Überprüfung der Vorgänge auf Richtigkeit potentiell jedermann offensteht (121ff.). Wegen drohender sozialer Folgen eines Protests ist das Ergebnis durch den von der Entscheidung Betroffenen als Prämisse seines weiteren Verhaltens zu übernehmen; alternativ drohen negative Sanktionen. Ziel ist die „Umstrukturierung von Rechtserwartungen“ (119), nicht die Umstrukturierung der personalen Umwelt in Form einer Überzeugung von der Richtigkeit des Urteils.

Äußerer Halt für diese Form der Übertragung reduzierter Komplexität ist aber, dass (rechtliche) Verfahren gesamtgesellschaftlich „generelle Anerkennung“ (31) erfahren. Ohne dieses „soziale Klima“ (34), das sich in der Moderne ausgebildet und an die Stelle ,naturrechtlicher Begründungen oder tauschförmiger Methoden der Konsensbildung“ tritt (30), wäre der Verweis auf die freiwillige, gleichberechtigte Schaffung einer Verfahrensgeschichte nicht anschlussfähig an die gesellschaftliche Umwelt.

Wird vor diesem theoretischen Hintergrund nach der Bedeutung professioneller Interpretationsleistungen und Aushandlungen auf der Hinterbühne gefragt, dann muss untersucht werden, (a) welcher Beitrag durch sie zur Reduktion fallbezogener Komplexität geleistet wird und (b) in welchem Verhältnis sie zur intersubjektiven Übertragung reduzierter Komplexität im formalen Verfahren stehen. Dabei müssen zwei Aspekte professionellen Handelns in den Fokus rücken, die Luhmann nicht systematisch in seine Analyse miteinbezieht:

(1) Der Laie muss vorbereitet werden auf seine Verfahrensrolle, selbst wenn dieser durch die Ungewissheit des Ausgangs motiviert ist, diese zu übernehmen. Erst ein Rechtsbeistand ermöglicht dem Laien, durch das Verfahren zu navigieren und damit die zur Legitimation des Ergebnisses zentrale Wahrnehmung des Grundrechts auf ein „faires Verfahren“ (Art. 6 EMRK). Hier ist die Frage, in welchem Verhältnis dies zum Mechanismus der freiwilligen Selbstfestlegung steht. 
(2) Weiterhin muss auch der Fall selbst, der vor Gericht verhandelt wird, vorbereitet sein. Der professionell konstruierte Fall ist bereits eine Form reduzierter Komplexität. Hier ist die Frage, in welchem Verhältnis dies zur Trichterfunktion des Verfahrens steht.

Um beide Aspekte bei der Durchführung einer empirischen Untersuchung systematisch berücksichtigen zu können, bedarf es einer den theoretischen Blick erweiternden professionssoziologischen Reflexion.

\section{Professionssoziologische Reflexion}

Werden Professionen in erster Näherung entlang traditioneller Ansätze betrachtet, lassen sie sich verstehen als ,exclusive occupational groups applying somewhat abstract knowledge to particular cases“ (Abbott 1988, 8). Ihre gesellschaftliche Funktion definiert sich durch die „Etablierung und Aufrechterhaltung kulturell gültiger Realitätsdefinitionen auf der Basis wissenschaftlichen Wissens“ (Klatetzki 2012, 168). Dabei besitzen sie das Mandat zur autonomen kompetenzbasierten Problembearbeitung, das sie nach dem Prinzip der Kollegialität prozessieren (Freidson 2001; Parsons 1947, 60). Durch die Transformation bzw. (Re-) Definition der Krisensituation des Laien in einen „Fall“ (Maiwald 2008) und der damit einhergehenden Reduktion von Komplexität auf spezifische Sinnzusammenhänge für die institutionalisierte Formen sozialer Organisation wie Gerichtsverfahren zur Problembearbeitung bereitstehen, führen sie alltagsweltliche Problemstellungen einer Lösung zu.

Dies kann jedoch nur gemeinsam mit dem Laien gelingen: Laien liefern nicht einem Automaten gleich die zur Fallkonstruktion und Bearbeitung notwendige Information und gliedern sich nicht ohne Weiteres in die standardisierten Prozeduren von Gerichtsverfahren ein. Reduktion von Komplexität durch die Transformation von Umweltkomplexität in einen Fall stellt damit ebenso wie die Schaffung einer Verfahrensgeschichte vor Gericht ein ko-produktives Verhältnis dar. Durch welche Strukturelemente sich ein solches „Arbeitsbündnis“ (Oevermann 1997, 152) konkret auszeichnet, ist zwar eine feldspezifische und empirische Frage, bedarf jedoch einer theoretischen Rahmung. Vor dem Hintergrund der Bezugnahme auf die theoretischen Grundlagen von „Legitimation durch Verfahren“ ist es dazu instruktiv, den von Kurtz (2011) rekonstruierten professionssoziologischen Ansatz Luhmanns ergänzend hinzuzuziehen.

In dieser Perspektive öffnen Professionen kommunikativ geschlossen operierende Funktionssysteme zur Umwelt (Kurtz 2011, 44). Funktionsäquivalent zu 
Organisationen, erbringen sie eine „Respezifikation von $\mathrm{zu}$ allgemein geratenen Kriterien“ (Luhmann 1997, 189) auf den Einzelfall. Professionen sind als „Form“ funktional, weil sie „den Entscheidungsspielraum in der Gesellschaft bzw. den Funktionssystemen der Gesellschaft einschränken.“ (Kurtz 2011, 44). Die hier notwendigen Entscheidungen darüber, was der Fall ist - und damit, was nicht der Fall ist - muss das Professionsmitglied kommunikativ in Aushandlung mit dem Laien herstellen. Dabei herrscht ein „Technologiedefizit“ $(2011,41)$ vor. Anders als im Verfahren stehen keine festen formellen Prozeduren bereit, wie die Ko-Produktion durchzuführen ist.

Ein besonderer Aspekt dieser „Arbeit an der Veränderung von Personen“ $(2011,47)$ ist, dass durch diese „Änderung [d]er personalen Umwelt“ auch „Inklusionsprobleme“ in institutionalisierte Formen sozialer Organisation wie Gerichtsverfahren bewältigt werden (2011, 41f.). Konkret wirkt professionelle juristische Kommunikation darauf hin, dass der Laie nicht nur an der Herstellung der Fallkonstruktion partizipiert, sondern diese auch als Verhaltensprämisse bei der Ausfüllung seiner Verfahrensrolle berücksichtigt. Auf die eine oder andere Weise müssen die Erwartungsstrukturen des Laien umgestellt werden, so dass dieser die Fallkonstruktion zumindest insoweit anerkennt, dass er diese bei seinem Rollenverhalten im Gerichtsverfahren berücksichtigt. Ob dies auch einen unabhängigen Beitrag zur intersubjektiven Übertragung reduzierter Komplexität leistet, bleibt eine empirisch offene Frage.

Wird zusätzlich das Prinzip der Kollegialität in diese Überlegung hinzugezogen, ergibt sich, dass der konstruierte Fall nicht nur gemeinsam mit dem Laien koproduziert und von diesem als Verhaltensprämisse übernommen werden muss, sondern zudem kollegialer Anerkennung bedarf. Der Fall bindet die professionellen Akteure aneinander, weil die Ausfüllung verfahrensbeteiligter Rollen die Anerkennung geteilter professioneller Standards impliziert. Professionsmitglieder teilen qua Ausbildung bereits eine Perspektive darüber, welche Formen der Reduktion fallbezogener Komplexität Gegenstand informeller Kooperation sein können und welche nicht; dies insbesondere in Hinblick auf die Art und Weise der dazugehörigen nichtöffentlichen Anwendung des Konditionalprogramms des Rechts. Informelle kollegiale Kommunikation kann nicht in beliebiger Form fallbezogene Komplexität reduzieren. Es ist folglich Teil der Reduktion fallbezogener Komplexität, dass im Kollegium in der Form gemeinsam Entscheidungen getroffen werden, dass der Fall geteilten wissenschaftlich-methodischen Standards genügt, um verhandelt werden zu können.

Durch das Prinzip der Kollegialität wird daher ebenso wie in der ExpertenLaien-Beziehung eine Respezifikationsleistung für den Einzelfall erbracht. Kollegiale Kommunikation ist dabei dadurch charakterisiert, dass sie nicht gleichzusetzen ist mit der öffentlich-rituellen Kommunikation der verfahrensbeteiligten 
Juristen in ihren jeweiligen Verfahrensrollen. Kollegiale Fallarbeit definiert sich gerade dadurch, dass sie durch ein hohes Maß an institutionalisierter Informalität gekennzeichnet ist, die von der formalen Verfahrensgeschichte abgekoppelt ist (Holzinger 2015; Scheffer/Hannken-Illjes/Kozin 2010). Ein klassisches Beispiel im Strafverfahren ist die informelle Institution des „Deals“ (Altenhain/Hagemeier/Haimerl/Stammen 2007; Altenhain/Dietmeier/May 2013).

Ist das Ziel vor dem theoretischen Hintergrund professionelle Kommunikation als Hinterbühne rekonstruiert, so dass sie mit den Mechanismen von Legitimation durch Verfahren ins Verhältnis gesetzt werden kann, lassen sich aus der Reflexion folgende analytische Ebenen ableiten, die als Heuristik einer empirischen Untersuchung des Strafverfahrens dienen können:

(1) Formen der Reduktion von (Umwelt-)Komplexität in einen vor Gericht verhandelbaren Fall durch das Prinzip kollegialer Fallbearbeitung und Ko-Produktion in der Experten-Laien-Beziehung (vgl. Abschnitt 6.a.).

(2) Formen der Arbeit an der personalen Umwelt zur Bewältigung von Inklusionsproblemen des Laien in seine Verfahrensrolle, insbesondere in Hinblick auf Mechanismen der (intersubjektiven) Übertragung reduzierter Komplexität, d.h. der Verhinderung der Institutionalisierung von Protest (vgl. Abschnitt 6.b.).

(3) Mechanismen der Institutionalisierung informell-professioneller Kommunikation (vgl. Abschnitt 6.c.).

Durch die Anwendung dieser Heuristik sollten sich folgende offene Fragen beantworten lassen, die das Verhältnis professioneller Kommunikation auf der Hinterbühne und den Mechanismen von Legitimation durch Verfahren auf der Vorderbühne zentral definieren (vgl. Abschnitt 7.):

(1) In welcher Form wird fallspezifische Komplexität durch kollegiale Fallarbeit reduziert und in welchem Verhältnis steht dies zu den Mechanismen der Reduktion von Komplexität und Übertragung reduzierter Komplexität durch das System des Strafverfahrens?

(2) Da in der Experten-Laien-Beziehung eine Realitätsdefinition ausgehandelt wird, auf welche Weise vollzieht sich dies konkret und in welchem Verhältnis steht dieser Prozess zur Reduktion von Komplexität und Übertragung reduzierter Komplexität durch das System des Strafverfahrens?

(3) Was sind Strukturen und Mechanismen, die es ermöglichen, dass sich Systeme professioneller Kommunikation auf der Hinterbühne reproduzieren? 


\section{Forschungsstand}

Obwohl der Ruf nach einem rechtssoziologischen Zugang, der die Erforschung der Erzeugungssituation von Recht in den Vordergrund stellt, in den letzten Jahren zunehmend lauter wird (Gephart 2012; Morlok/Kölbel/Launhardt 2000; Morlok/Kölbel 2001; Müller-Mall 2012; Rensen 2017; Stegmaier 2013; Wrase 2016; 2017 a; 2017 b), geben bisherige empirische rechtssoziologische Studien über die erarbeitete Fragestellung nur unzureichend Auskunft.

Zunächst deswegen, weil sich bisherige Untersuchungen vorwiegend mit dem Entscheidungsprozess bzw. der Praxis Richtender beschäftigt haben (aktuell Kranenpohl 2010; Latour 2010; Stegmaier 2009; klassisch Lautmann 1970; 1972). Dies gibt nur eingeschränkt Auskunft über Kommunikation in Interaktion mit Laien und anderen Professionsmitgliedern auf der Hinterbühne, da lediglich die Perspektive Richtender rekonstruiert wird.

Andererseits konzentriert sich eine Vielzahl von Einzelstudien auf die Rekonstruktion der Vorderbühne mündlicher Verhandlung, was ebenso keinen Rückschluss auf die Hinterbühne und die dort stattfindende professionelle Kommunikation zulässt (z. B. Hannken-Illjes 2006; Hoffmann 1983; 2010; Legnaro/ Aengenheister 1999; Reichertz 1984; Schütze 1978; Seibert 2004).

Schließlich wird, wenn professionssoziologisch die Experten-Laien-Beziehung in den Fokus rückt (Eidmann 1994; Wernet 1997), nur unzureichend eine systematische Verbindung zwischen Experten-Laien-Beziehung und kollegialer Fallbearbeitung hergestellt.

Als Folge besteht weder über die Praxis von Staats- und Rechtsanwälten noch über die (informelle) Kommunikation der Professionsmitglieder untereinander fundiertes Wissen (zu diesem Defizit Baer 2015, 172; Rehbinder 2014, $153 \mathrm{ff}$.).

Hervorzuheben ist in diesem Zusammenhang die Studie von Scheffer/ Hannken-Illjes/Kozin (2010), die ebenso an den Verfahrensbegriff von Luhmann anknüpft (Scheffer 2010), auf Kommunikationsstrukturen der Vorderbühne ausgerichtet ist, aber auch informell-professionelle Kommunikation empirisch in den Blick nimmt. In Bezug auf das deutsche Strafverfahren wird dabei eine umfassende Bedeutung informeller Kommunikation für die Verfahrenspraxis stark relativiert. Besonders Absprachepraktiken seien nicht die Regel und würden dies auch in Zukunft nicht sein, denn sie bedrohten die Vormachtstellung des Richters in seinem Selbstverständnis als zentral mit der Fallaufklärung betrauten Rolle (Scheffer/Hannken-Illjes/Kozin 2010, 162).

Diese These, in der die Formalitäts-Informalitäts-Spanne in Richtung der Prädominanz der Befolgung formaler Verfahrensrollen ausschlägt, kann aber nicht nur theoretisch bezweifelt werden (Holzinger 2015). Sie erscheint auch vor dem Hintergrund der Beschäftigung des Bundesverfassungsgerichts mit der Abspra- 
chepraxis in deutschen Strafverfahren und der dazugehörigen fragebogenbasierten Studie (Altenhain/Dietmeier/May 2013) fraglich. Letztere beinhaltet Indizien dafür, dass informelle Absprachen unter verfahrensbeteiligten juristischen Akteuren weit verbreitet sind (2013, $53 \mathrm{ff}$.).

Zusammengefasst bleibt es also eine empirisch offene Frage, in welchem Verhältnis formale Mechanismen der Reduktion und Übertragung von fallbezogener Komplexität zu informell-professioneller Kommunikation stehen. Darüber hinaus fehlt es in Bezug auf das deutsche Strafverfahren vor dem Hintergrund der Formalisierung der Verfahrensabsprache durch „Das Gesetz zur Regelung der Verständigung im Strafverfahren“ im Jahre 2009 an einem qualitativen Zugriff, der sich damit befasst, wie sich informell-professionelle Kommunikation unter den geänderten rechtlichen Vorzeichen des eingeführten § 257c StPO faktisch vollzieht.

\section{Datengrundlage und Methode}

Die Datengrundlage besteht maßgeblich aus 25 problemzentrierten Interviews (Witzel 2000) mit an Strafverfahren beteiligten Juristen. Innerhalb eines „[s]elektiven Samplings“ (Schatzman/Strauss 1973, 38 ff.) entlang verfahrensbeteiligter Rollen wurde ein „theoretisches Sampling“ (Corbin/Strauss 1990, 420 f.) durchgeführt. In das Sample aufgenommen wurden vier Amtsrichter, zwei Richter am Landgericht, zwei vorsitzende Richter am Landgericht, sechs Staatsanwälte, drei Oberstaatsanwälte sowie acht Rechtsanwälte, die einen Schwerpunkt auf Strafverfahren aufwiesen. Vier der Staatsanwälte waren Teil einer auf Wirtschaftskriminalität spezialisierten Staatsanwaltschaft, drei der Rechtanwälte waren Fachanwalt für Strafrecht. Die juristischen Akteure wurden als Experten ihrer eigenen Wirklichkeit behandelt (Bogner 2005; Gläser/Laudel 2009; Przyborski/WohlrabSahr 2014, 119 ff.). Den Problemhorizont bildete die individuelle und kollegiale Fallbearbeitung sowie die Arbeit mit verfahrensbeteiligten Laien. Darüber hinaus wurden 15 Sitzungstage an Amts- und Landgerichten teilnehmend beobachtet. Die Daten wurden ursprünglich im Rahmen eines Forschungsvorhabens über die juristische Fallbearbeitung in Straf- und Zivilverfahren gewonnen (Starystach 2018) und zur Beantwortung der aufgeworfenen Fragestellung sekundär ausgewertet. Auf Basis der verwendeten Daten kann die Fragestellung nur in Hinblick auf das Strafverfahren und hier auch nur in Bezug auf die Instanzen des Amts- und Landgerichts beantwortet werden. Weitere Limitation ist, dass ein qualitatives Forschungsdesign zwar ermöglicht, typische Formen informell-professioneller Kommunikation herauszuarbeiten, aber es kann auf diesem Weg keine Auskunft über den Verbreitungsgrad dieser Typen im statistischen Sinne gegeben werden. 
Vor dem Hintergrund des Erkenntnisinteresses wurde in der Analyse die Rekonstruktion kollektiv geteilter Vorräte an „Gebrauchs-“ bzw. „Rezeptwissen“ (Schütz/Luckmann 2003, 139 ff.) ins Zentrum gestellt. Es wurden diejenigen kognitiven Schemata herausgearbeitet, die es ermöglichen, in der Fallbearbeitung auftretende Kontingenz zu bewältigen. Dazu wurde das Datenmaterial mit Hilfe einer explizierend-strukturierenden Inhaltsanalyse ausgewertet (Mayring 2015).

Konkret wurden (1) in den Interviews alle narrativen Sequenzen identifiziert, in welchen (a) juristische Akteure über ihre Fallarbeit berichteten oder (b) über die Ausfüllung ihrer Rolle als prozessbeteiligter Jurist im Kontext des Strafverfahrens reflektierten. Darauf aufbauend wurden (2) sämtliche identifizierten Sequenzen in-vivo codiert und anschließend paraphrasiert. Innerhalb dieses inhaltanalytisch strukturierten Korpus diente (3) ein „synoptischer Vergleich“ (Sachweh 2010, 128) dazu, intersubjektiv geteilte informelle Kommunikationsstrukturen in der juristischen Fallarbeit zu rekonstruieren. Da die Daten an unterschiedlichen prozessbeteiligten Rollen erhoben wurden, konnten (4) die jeweiligen Bestände an kollektiv geteiltem Gebrauchs- und Rezeptwissen zum Zwecke der Objektivierung trianguliert werden (Flick 2008). Eine weitere Triangulation bestand darin, die kognitiven Muster ins Verhältnis zu Interaktion professioneller Akteure in mündlichen Verhandlungen zu setzen. Abschließend wurden (5) die systematisierten Sinngehalte auf die dargelegten analytischen Ebenen bezogen, um die Ergebnisse mit Hilfe des theoretischen Rahmens in einen funktionalen Gesamtzusammenhang zu stellen. Entsprechend orientiert sich die Darstellung an den unter Abschnitt 3. dargestellten analytischen Dimensionen. Zum Zwecke der Nachvollziehbarkeit werden solche Interviewpassagen eingeführt, die als Ankerbeispiele für die in-vivo Codierung gedient haben.

\section{Die Funktion informeller Kommunikation im Kontext des deutschen Strafverfahrens}

\section{6.a. Reduktion von fallbezogener Komplexität}

Mit der Reduktion von fallbezogener Komplexität im Strafverfahren ist im typischen Fall zunächst die Organisation der Staatsanwaltschaft betraut. Bereits reduzierte Komplexität in Form polizeilicher Akten muss in rechtliche Sachverhalte transformiert werden. Auf Basis der Ermittlungen der Organisation der Polizei ist darüber zu entscheiden, ob eine Anklage erhoben wird, das Verfahren damit von der Phase des Ermittlungsverfahrens in diejenige des Zwischen- 
verfahrens eintritt, d.h. in den Verantwortungsbereich des Richters übergeht, oder (gegen Auflage) eingestellt wird. Externe Realitätsdefinitionen werden für die Anwendung rechtlicher Kategorien aufbereitet und damit objektiviert. Eine Tätigkeit, die sich grundlegend von der in der Universität eingeübten juristischen Methodenlehre unterscheidet. Die Subsumtion selbst, das heißt die Anwendung des juristischen Kategoriensystems, ist nicht das zentral bearbeitete Problem, sondern die Reduktion von Umweltkomplexität in subsumierbare Sachverhalte:

„Wenn man von der Uni kommt, kriegt man diese vorbereiteten Sachverhalte, die einen Absatz lang sind. [...] Und dann muss man halt unglaublich auslegen, wie ist das gemeint. In der Praxis ist das anders. Da kriegen Sie halt unglaubliche Papiermengen und müssen da irgendwas daraus machen. Und das ist halt ein totaler Paradigmenwechsel. Das hat einen als Referendar völlig umgehauen. [...] Aber das ist, glaube ich, einfach nur eine Frage der Gewöhnung letzten Endes, wie man, dass man das Wesentliche da rausfiltern kann.“ (S7 37) ${ }^{7}$

Fälle werden nach Beweisbarkeit sortiert. Diejenigen, die umfangreichen Ermittlungen bedürfen, werden eingestellt oder anhand der Aktenlage auf eindeutig beweisbare Sachverhalte reduziert, die erfolgreich angeklagt werden können. In welcher Form die Arbeit des Filterns erbracht wird, hängt folglich nicht nur von der Fallkonstellation, sondern auch von der Zeitökonomie ab:

„Wenn man viel zu tun hat, natürlich neigt man eher dazu zu sagen, ich begrenze mich auf die Anzeige, was angezeigt ist. Wenn ich weniger zu tun habe, [...] dann steige ich halt tiefer ein. [D]as kann man schon steuern.“ (S5 47)

Vor diesem Hintergrund werden Nachermittlungen außer in Einzelfällen der Polizei überlassen. Direkter Kontakt zur Komplexität der Alltagswelt, insbesondere zu Beschuldigten und Zeugen, wird vermieden.

Die Arbeit des Filterns, insbesondere „tieferes Einsteigen“ in einen Fall, muss sich „rentieren“ (S5 45). Das Prinzip der Rentabilität vermittelt zwischen dem organisationalen Anspruch eingehende Fälle effizient abzuarbeiten und rechtsstaatlichen Grundsätzen. Vor dem Hintergrund der Output-Orientierung der bürokratischen Organisation naheliegende Formen „brauchbarer Illegalität“ (Luhmann 1964) - insbesondere in Form der Einstellung möglichst vieler Verfahren durch die Instrumentalisierung professioneller Entscheidungsautonomie werden durch professionelle Standards eingeschränkt, die mit der Herstellung gesellschaftlicher Anerkennung von Verfahren verbunden sind. Entsprechend

7 Zur Nomenklatur: „Sx“ bezeichnet Belegstellen aus Interviews mit Staatsanwälten, „RAx“ mit Rechtsanwälten und „Rx“ mit Richtern. Die darauffolgende Zahl zeigt die Zeile des Interviewtranskripts an, an der die Belegstelle beginnt. 
werden z. B. schwere Straftaten - genannt werden stets Mord, Totschlag, Sexualdelikte - stets ausermittelt und selbst wenn eine Verurteilung nicht sicher erscheint, angeklagt. Ein Verweis auf zeitökonomische Ressourcen ist hier nicht zu rechtfertigen.

Die zum Filtern notwendigen „Vergleichsmaßstäbe“ (S5 167) bilden sich im kollegialen Austausch mit impliziten Verpflichtungscharakter aus. Zentrale Manifestation ist die „Kaffeerunde“ (S1 8; S3 52; S5 163; S6 124; S8 9):

„Und wenn einer ein Problem hat oder weiß nicht, was er in dem Fall machen soll, dann geht er halt mal zum Kollegen und bespricht das. Oder es ist eine Kaffeerunde in der Woche und dann trägt der das vor. Oder er kommt auch rüber, wenn mehrere Tage Verhandlung waren und erzählt dann was war und fragt nach einem Rat, gerade was Strafzumessung betrifft, kann man auch mal diskutieren. Also das wird hier recht offen kommuniziert. Zumindest bei mir in der Abteilung gibt es keinen Kollegen, der so ein Einzelgänger ist oder ein Eigenbrötler.“ (S3 48)

Auf diese Weise bildet sich innerhalb der Staatsanwaltschaft ein informeller Korridor aus, in dem kollegial respezifiziert wird, ob und in welcher Form zugewiesene Komplexität weiter in einen verhandelbaren Fall reduziert wird.

Bei Anklage werden dem Richter die „Tatsachen auf den Tisch“ (S6 141; S2 17) gelegt und damit das Zwischenverfahren eingeleitet. „Tatsachen“ ist wörtlich zu nehmen,

„weil normalerweise haben [Richter] die Erwartung, wenn wir was anklagen, dass die Dinge auch verurteilt werden. Wir stellen ja $70 \%$ der Verfahren ein. Sagt unser Chef immer. Ich habe die Zahlen nicht überprüft, ist aber wohl so. Deswegen ist natürlich die Erwartung so, wenn wir was anklagen, dass es halt auch hält.“ (S7 52)

Da die durch Filtern auf einen Fall reduzierte Umweltkomplexität in Form von Sachverhalten nun dem Konditionalprogramm des Rechts gehorcht und unter dem Prinzip der Rentabilität zustande kam, erscheint im typischen Fall auch aus der Perspektive der richterlichen Rolle ein Zweifel an der Richtigkeit dieser Anordnung subsumierbarer Sachverhalte für den weiteren Verfahrensverlauf dysfunktional. Anklagen werden typischerweise als gültige Realitätsdefinitionen anerkannt:

„Staatsanwälte sind objektiv, die stellen wesentlich mehr Verfahren ein, als sie anklagen. Also, sie müssen ja allem nachgehen und wenn was zur Anklage kommt, dann spricht schon viel dafür, dass sie da keinen Unschuldigen da sitzen haben, so möchte ich es mal sagen. [A]lso ich spreche einmal im Jahr durchschnittlich einen frei. Nicht weil der unschuldig wäre, sondern ganz einfach es ist so, dass die Beweislage so ist, dass ich nicht revisionssicher verurteilen kann.“ (R10 106) 
Dieses Vertrauen der Richterschaft in die Gültigkeit staatsanwaltschaftlicher Realitätsdefinition ist dabei gerade nicht von informeller Kommunikation gestützt. Staatsanwälte reichen Fälle zur Bearbeitung weiter. Außer im Falle von Ermittlungsrichtern gibt es vor der mündlichen Verhandlung typisch keine informelle kollegiale Fallbearbeitung. Staatsanwälte informieren Richter jedoch gelegentlich, wenn es sich um einen Fall handelt, der angeklagt wird, dessen Verurteilung jedoch nicht gesichert erscheint, aufgrund staatsanwaltsinterner „Vergleichsmaßstäbe“ dennoch anzuklagen ist. Ursache dafür liegt im Organisationscharakter der Staatsanwaltschaft begründet. Die Anklage ist die offiziell dokumentierte Position der Organisation der Staatsanwaltschaft, die, ist sie einmal in der Welt, formale Selbstfestlegung erzeugt. Zusätzlich ist die Teilnahme an der mündlichen Verhandlung getrennt von der Fallbearbeitung. In der Folge garantiert eine erfolgreiche Abstimmung zwischen Fallbearbeiter und Richter im Ermittlungs- und Zwischenverfahren keine erfolgreiche Kommunikation mit dem Sitzungsteilnehmer. Die Zweckprogrammierung und hierarchischarbeitsteilige Struktur der Arbeitsorganisation setzt professioneller Autonomie und damit dem Gegenstandsbereich informell-professioneller Kommunikation Grenzen.

Da im prototypischen Verfahrensverlauf durch Richtende an den aktenförmig objektivierten Sachverhalten nicht gezweifelt wird, ist das zentral bearbeitete Problem, die mündliche Verhandlung vorzubereiten. Dazu suchen Richter nur im Einzelfall Austausch mit Kollegen. Systematische informelle Kommunikation wie in der Staatsanwaltschaft besteht in der Richterschaft nicht. Strafrichter betonen in besonderem Umfang ihre richterliche Unabhängigkeit, da diese ihnen ermöglicht, frei innerhalb der als gültig anerkannten Realitätsdefinition vor dem Hintergrund, was die Konditionalprogramme des Strafrechts und der StPO vorgeben, ein Skript für die mündliche Verhandlung zu erstellen.

Anhand der durch die Anklage strukturierten Akte wird die mündliche Verhandlung so geskriptet, dass die notwendige Einführung von Beweisen, Befragung von Zeugen und die Urteilsverkündung zeitökonomisch effizient und gegen Aufhebung geschützt vollzogen werden kann. Dass dieses Skript aber seine Funktion erfüllen kann, ist abhängig vom Verhalten anderer Verfahrensbeteiligter, die das Recht haben, eingeführte Beweise in ihrer Gültigkeit durch die formalen Mittel der StPO in Frage zu stellen. Das Prinzip, das es ermöglicht, diese Komplexitätsreduktion erfolgreich zu vollziehen, ist dasjenige der Zufriedenheit:

„Eine gute Verhandlung macht für mich aus, [w]enn alle zufrieden sind. [...] Wenn man ein Ergebnis hat, mit dem alle leben können, ja? Wenn es nur um das Strafmaß geht ja und der - was weiß denn ich - der Angeklagte sagt, mit zwei sechs wäre mir gedient, dann habe ich die und die Möglichkeiten ja, und der Staatsanwalt sagt, ich hätte aber lieber drei Jahre 
und man sagt, mit zwei acht können sie alle leben irgendwo noch. Dass man da also so einen Kompromiss findet, mit dem alle leben können, weil Sie können zwei sechs verhängen oder drei, es gibt keine Punktstrafe, ja.“ (R9 101)

Zentral findet das Prinzip der Zufriedenheit Anwendung in der Beziehung zum Angeklagten. Die Staatsanwaltschaft kann zwar ebenso mit formalen Mitteln den geskripteten Verlauf der mündlichen Verhandlung bedrohen, ist aber aufgrund der Überlastung dieser Organisation, die sich in minimal vorbereiteten Sitzungsteilnehmern niederschlägt, auch durch richterliche Unabhängigkeit in der Entscheidungsfindung in mündlichen Verhandlungen leichter zu kontrollieren.

Als Gegenleistung für die Mitwirkung wird dem Angeklagten innerhalb des durch das Konditionalprogramm des Strafrechts implizierten Strafrahmens Strafnachlass geboten. Diese Instrumentalisierung des Konditionalprogramms des Rechts schafft die Bedingung der Möglichkeit dafür, das Inklusionsproblem zu lösen, grenzt dadurch aber zwangsläufig formal über den Strafrahmen ein, was informell verhandelbar ist und was nicht. Die Aufgabe des Verteidigers ist es, hier die entsprechende Vermittlungsleistung und im Falle einer streitigen Verhandlung notwendige Informationen zur Vervollständigung des Skripts zu erbringen:

„Was heißt kooperativ?! Es gibt Verteidiger, die von vornherein sich mit den Mandaten überlegen: Was ist unser Ziel? Wenn das Ziel ist, eine möglichst geringe Strafe, also, wenn der Mandant dann sagt: Ja, ich habe das begangen, dann kommen die von vornherein auch mit einem Geständnis und arbeiten dann auf eine möglichst milde Strafe hin. Auch wenn die der Meinung sind, sie müssen jetzt bestreiten, dann sagen sie frühzeitig, welche Beweise aus ihrer Sicht nötig sind [...] und dann kann man den Prozess entsprechend gestalten und dann arbeiten sie mit und finden sich dann halt mit dem ab, was das Gericht dann macht. Nehmen sozusagen konstruktiv Einfluss.“ (R12 360)

Diese informelle Kommunikation zwischen Verteidigung und Richterschaft dient dazu, die mündliche Verhandlung in ihrem Verlauf vorzubereiten. Dabei wird auch von Rechtsanwälten die staatsanwaltschaftliche Realitätsdefinition als Grundlage des mündlichen Verfahrens im typischen Fall anerkannt:

„Die Staatsanwaltschaft prüft schon sehr genau, bevor sie Leute beschuldigt oder gar anklagt. [...] Also wenn die weiterverfolgen, dann wissen die in der Regel schon, warum.“ (RA12 10)

Vor diesem Hintergrund besteht das zentral durch den Verteidiger zu bewältigende Problem im typischen Fall darin, bei der Reduktion der Aktenlage in ein Skript der mündlichen Verhandlung affirmativ-konstruktiv mitzuwirken. Im Ermittlungsverfahren erfüllt die Verteidigung aufgrund der hohen Entscheidungsautonomie der Staatsanwaltschaft eine ähnliche Vermittlungsfunktion, 
wobei aber umfangreichere Einflussmöglichkeiten auf die Realitätsdefinition in Form von rechtlich subsumierbaren Sachverhalten bestehen:

\begin{abstract}
„Man kann natürlich auch schreiben, aber manchmal spricht es sich am Telefon auch leichter und schneller. Passiert dann. Dann ruft man an und sagt, wie sieht das denn aus, Sie haben ja gesehen, die Ehefrau meines Mandanten, die ihn hier jetzt anzeigt, die hat ja auch kräftig mitgemischt, die war ja noch ganz aufgebracht, als die Polizeibeamten kamen und ist auf die selbst fast losgegangen, kann man das nicht einstellen. Körperverletzung im häuslichen Bereich, da gibt es doch kein öffentliches Interesse, die sind jetzt sowieso getrennt lebend, die werden demnächst geschieden, können wir da nicht eine Einstellung machen. Und dann spricht man darüber und dann findet man eine Lösung.“ (RA13 21)
\end{abstract}

Fluchtpunkt einer Verteidigungsstrategie ist es, bereits im Ermittlungsverfahren in kollegialer Fallarbeit mit der Staatsanwaltschaft Einfluss auf die Konstruktion der Akte zu nehmen und im besten Falle durch eine Einstellung „den Deckel so früh wie möglich drauf zu machen, logischerweise“ (RA16 14). Gespräche mit der Staatsanwaltschaft werden im typischen Fall sehr offen geführt. Dabei können auch „Gesamtlösungen“ (S3 122) für umfangreich straffällig Gewordene „kollegial“" (S7 74) diskutiert werden.

Beide Dyaden Staatsanwaltschaft-Verteidigung sowie Richterschaft-Verteidigung haben eindeutig informellen Charakter, sie erfolgen in der Regel telefonisch, so dass keine formalen Selbstfestlegungen entstehen. Vor diesem Hintergrund sind für die Arbeit eines Verteidigers „Kontaktsysteme“ (Luhmann 1983, $75 \mathrm{ff}$.) zur Staatsanwaltschaft und der Richterschaft zentral, da sie notwendiges Vertrauen für informelle Kommunikation bereitstellen. Je umfangreicher die Kontaktsysteme, desto weniger ist auch die Berücksichtigung des formalen Rahmens des § 257c StPO notwendig. Insbesondere kann auch der nicht mehr gestattete Rechtsmittelverzicht vereinbart werden und darüber hinaus die Absprache durch wechselseitige Antizipation informeller Erwartungen im Impliziten verbleiben. Durch wiederholte Interaktion geschaffene Kontaktsysteme ermöglichen also, den Gegenstandsbereich desjenigen, was informell verhandelbar ist, zu vergrößern und damit die Differenzierung zwischen Vorder- und Hinterbühne auszuweiten.

Die Reduktion von Komplexität durch die Profession ist ein mehrstufiger Prozess der Respezifikation von zu allgemein geratenen Kriterien des Konditionalprogramms des Strafrechts und insbesondere der StPO. Dieser Prozess kann für typische Fälle in seinem Verlauf wie folgt rekonstruiert werden: Zunächst werden der Organisation der Staatsanwaltschaft zugewiesene Fälle gefiltert, d.h. in subsumierbare Sachverhalte transformiert, die dem Konditionalprogramm des Rechts gehorchen. Dabei findet das Prinzip der Rentabilität Anwendung, das aus „Vergleichsmaßstäben“ besteht, die zwischen der organisational bedingten Anforderung einer effizienten Bearbeitung von Umweltkomplexität auf der 
einen und rechtstaatlichen Prinzipien auf der anderen Seite vermitteln. Dabei kann die Erstellung dieser Realitätsdefinition in einem begrenzten Rahmen durch den Verteidiger nach dem Prinzip der Kollegialität beeinflusst werden, daran anschließend besteht dessen Aufgabe in der Vermittlung der ausgehandelten Realitätsdefinition und damit verknüpfter Angebote der Staatsanwaltschaft an den Mandanten. Ist die staatsanwaltliche Realitätsdefinition jedoch in Form einer Anklage an das Gericht übergeben worden, erlangt sie im typischen Fall umfangreiche Gültigkeit. In der Folge reduziert der Richter diese Komplexität weiter, indem er sie in ein Skript für eine mündliche Verhandlung überführt. Bei der Herstellung dieses Skripts orientiert er sich zentral an dem Prinzip der Zufriedenheit, um mögliche Infragestellung an der etablierten Realitätsdefinition in der mündlichen Verhandlung durch den Angeklagten unter Berufung auf formale Mittel der StPO auszuschließen. Hier vermittelt erneut der Verteidiger, jedoch mit einem stärkeren Schwerpunkt auf der Aushandlung der Bedingungen, unter denen die Realitätsdefinition durch das Verhalten der Verteidigung in der mündlichen Verhandlung mitgetragen wird. Die hier notwendige Arbeit an der personalen Umwelt ist im typischen Verlauf eines Strafverfahrens an die Experten-Laien-Beziehung zwischen Verteidiger und Mandant externalisiert.

\section{6.b. Lösung von Inklusionsproblemen in das Verfahrens- system durch professionelle Arbeit an der personalen Umwelt}

Die im Strafverfahren spätestens mit der Erhebung der Anklage etablierten Realitätsdefinition ist an den Mandanten dergestalt zu vermitteln, dass dieser seine Verfahrensrolle entsprechend des implizit antizipierten oder explizit festgelegten Skripts der mündlichen Verhandlung übernimmt. Die Rolle des Vermittlers zwischen „rechtsunverständigen Bürger“ (RA12 20) und der Justiz ist dabei auch im Selbstverständnis von Verteidigern institutionalisiert:

„Also meine Hauptarbeit ist die mit den Mandanten, um denen klarzumachen, hör mal, das ist nicht so, wie du denkst. Wenn du das und das machst, dann ist das bei verständiger Betrachtung, könnte es eine Straftat sein - also ich formuliere das jetzt vorsichtig also meine Hauptaufgabe besteht eigentlich darin, die Beschuldigten auf das einzustellen, was sie von Seiten der Justiz erwartet. Ich bin so das Bindeglied quasi. Ich bin der, der von seinem Auftrag her im Lager der Beschuldigten steht, aber von seiner Bildung her, dem juristischen Betrieb, dem juristischen Zirkus, recht nahesteht und da versuche, irgendwie die Enden zusammen zu bringen, die zunächst einmal nicht unbedingt zusammenpassen.“ (RA12 18) 
In der Experten-Laien-Beziehung ist aus funktionaler Perspektive die kollegial reduzierte Komplexität in Bezug auf ihre formalen Implikationen vor dem Hintergrund des Konditionalprogramms des Rechts anschlussfähig zu machen, um Compliance beim Laien zu erzeugen. Wird diese Verhaltensprämisse anerkannt, kann anschließend in Ko-Produktion eine Verteidigungsstrategie entworfen werden, die sich in das in der Richter-Verteidiger-Dyade erzeugte Skript der mündlichen Verhandlung funktional einfügt. Bei der Aushandlung der Rahmenbedingungen einer entsprechenden Übernahme der Verfahrensrolle muss gegenüber der Justiz das Gleichgewicht gehalten werden zwischen der Preisgabe von Informationen zur Weiterentwicklung bzw. Konsolidierung des Skripts - d.h. Reduktion von Komplexität - und erhaltener Gegenleistung in Form eines „Rabatts“ (R13 106) auf den im Gerichtsbezirk oder in der betreffenden Staatsanwaltschaft üblichen „Tarif“ (RA12 39; RA16 40). Gelingt dies, kann die Krisensituation des Laien überwunden werden, indem durch seine Inklusion in die Verfahrensrolle sein Interesse an einer geringen Strafhöhe entsprochen wird. Dass dieses Interesse durch kollegiale Reduktion von Komplexität erst im System des Strafverfahrens in seiner spezifischen Form entstanden ist, wird verdeckt.

Im typischen Fall ist das zentrale Prinzip bei der Arbeit an der personalen Umwelt dasjenige der Konfrontation des Mandanten mit der Realitätsdefinition der Justiz. Der Mandant ist damit vertraut zu machen, wo die „Knackpunkte“ (RA11 51) liegen, worauf durch die binnenrationale Übernahme der Verfahrensrolle noch Einfluss genommen werden kann und worauf nicht. Nur in Ausnahmefällen werden durch die Beisteuerung von Umweltkomplexität entlastende rechtlich subsumierbare Sachverhalte ko-produziert. Dieses systematische „Erwartungsmanagement“ (RA11 68) strukturiert den Raum des Rationalen und des Irrrationalen in der Ausfüllung der Verfahrensrolle durch den Laien:

\footnotetext{
„[W]ir sind schon dazu da [...], die Leute zu beraten und aufzuklären und vielleicht darauf hinzuwirken, dass sie ein für sich günstiges Verteidigungsverhalten an den Tag legen, aber ich werde niemanden zu einem Geständnis prügeln. Der danach sagt, mein Anwalt! [...] Das wäre dann möglicherweise auch ein falsches Geständnis, da wollen wir nicht drauf hinwirken. Das gibt es aber. In der Regel ist es wichtig, [...] dem Mandanten das Gefühl zu geben, dass er in der Situation, unabhängig davon, dass ich ihm das sage, wie es sich jetzt in der Akte darstellt, mich an seiner Seite weiß.“ (RA13 14)
}

Besondere Überzeugungskraft kann Konfrontation dadurch entfalten, dass der Verteidiger systematisch darauf verweist, dass es sich bei der Realitätsdefinition der Justiz um eine Krisensituation handelt, auf dessen Prämissen der Verteidiger selbst nur einen sehr begrenzten Einfluss hat. Durch diese Externalisierung kann diesem keine Verantwortung für den antizipierten Verfahrensverlauf zugeschrieben werden und dies gibt der Kommunikation äußeren Halt. 
In der Experten-Laien-Beziehung wird kommunikativ verdeckt, dass der Verteidiger innerhalb informeller professioneller Kommunikationsstrukturen an der Etablierung und Aufrechterhaltung dieser Realitätsdefinition, wenn auch nicht notwendig intentional, so zumindest funktional beteiligt ist. Der Verteidiger ist eben nur der Vermittler einer Situation, in der sich der Mandant für oder gegen rationales Verhalten entscheiden kann. Bindet sich der Mandant auf diese Weise freiwillig an die Ausfüllung seiner Verfahrensrolle, erzeugt er in Ko-Produktion eine Verteidigungsstrategie. Eine etablierte Verteidigungsstrategie, die sich in das Skript der mündlichen Verhandlung funktional einfügt, also die Lösung des Inklusionsproblems darstellt, wird im Anschluss dauerhaft durch den Verteidiger kommunikativ aufrechterhalten. Nachträglich aufkommender Protest wird unterbunden, um den Mandanten ,unter Umständen auch vor sich selbst zu beschützen“ (RA12 72):

„[W]enn es gut läuft, dann muss ich gar nicht groß was machen. Dann hat die Vorbereitung so gefruchtet, dass er sein Verslein aufsagt. [...] Dann gibt es die Fälle, wo man dem Mandanten dann schon mal zuflüstert, das jetzt besser nicht so sagen oder vergessen sie nicht sich zu entschuldigen beim Zeugen, Geschädigten. Und dann gibt es die ganz krassen Fälle, wo ich dann die Hand hebe und sage, Herr Vorsitzender jetzt brauche ich doch nochmal kurz eine Pause, schnappe mir meinen Mandanten und gehe mit dem raus und sage, hören Sie, das haben wir so nicht besprochen, wir müssen jetzt mal die Kurve kriegen, ja?! Möglichst bestimmt sein. Ja! [...] Also neben dem Mandanten zu sitzen und irgendwie zu träumen, ist keine gute Idee.“ (RA15 41)

Die Arbeit an der personalen Umwelt ist damit gekennzeichnet durch eine Umstrukturierung der Erwartungsstrukturen des Laien durch die Konfrontation mit der etablierten Realitätsdefinition in Form der Akte bzw. der Anklage. Innerhalb dieser Realitätsdefinition kann der Mandant sich entscheiden, seine Verfahrensrolle rational oder irrational in Bezug auf die durch seinen Verteidiger explizierten Interessen auszufüllen. Die Ursache für die jeweilige Manifestation dieser Realität wird der Justiz zugeschrieben und damit begründungstechnisch externalisiert. Die informelle Mitwirkung des Verteidigers wird auf diese Weise verdeckt und gibt der Konfrontation als Kommunikationsstrategie äußeren Halt. Im Falle des nun naheliegenden rationalen Verteidigungsverhaltens kann in KoProduktion eine entsprechende Verteidigungsstrategie entworfen werden, die das in der Richter-Verteidiger-Dyade kollegial konstruierte Skript für die mündliche Verhandlung konsolidiert:

Richter: „Und wie sieht es momentan mit dem Drogenkonsum bei Ihnen aus?”

Der Angeklagte führt aus, dass er als Gerüstbauer in „einem der zehn gefährlichsten Berufe“ arbeite und es deswegen „gar nicht geht“, Drogen zu konsumieren. Er gibt weiterhin an, dass er in den letzten 6 Monaten nach der Hausdurchsuchung in seiner Wohnung freiwil- 
lig und auf eigene Kosten „Drogenscreenings” durchgeführt habe; aber nur drei, weil sein damaliges Gehalt als Lehrling keine weiteren zugelassen habe.

Die Richter nimmt sich mit ostentativem Wohlwollen der Screenings, des Gesellenbriefs sowie des Arbeitsvertrages an. Der Richter erklärt, dass es ihm sehr zusage, was „vorgetragen” und „eingeführt” wurde, da es auf eine gute Entwicklung schließen ließe.

Der Angeklagte fügt hinzu, dass er in Zukunft „weiter ein verantwortungsvolles Leben führen möchte“ und die Hausdurchsuchung ein „Weckruf” für ihn gewesen sei, insbesondere sei ihm vor seiner neuen Freundin, mit welcher er zu diesem Zeitpunkt gerade zusammengezogen sei, der Beginn der Verhandlung sehr unangenehm gewesen, weil er eigentlich mit dieser „Phase seines Lebens” abgeschlossen habe und zurzeit auch ein duales Studium absolviere.

Richter: „Noch Fragen?”

Der Verteidiger hebt die Hand. Richter: „Na?!”, Verteidiger: „Nur um das abzurunden, möchte der Angeklagte zu Protokoll geben, dass er weitere Drogenscreenings - als Auflage auch auf eigene Kosten durchführen würde.”

(Amtsgericht, Verstoß gegen das Betäubungsmittelgesetz)

Durch das erfolgreiche Ineinandergreifen kollegialer Fallbearbeitung in den dargestellten Konstellationen in Kombination mit der kommunikativ abgekoppelten konfrontativen Vermittlung der auf diese Weise erzeugten Realitätsdefinition in der Experten-Laien-Beziehung, kann im typischen Fallverlauf die Durchführung der formal notwendigen mündlichen Verhandlung gesichert werden. Der Erfolg der Konfrontation wird durch Extermalisierung gesichert, indem von Seiten der Verteidigung die Verantwortung für die jeweilige Realitätsdefinition der Justiz zugeschrieben wird.

Dieser Inklusionsprozess auf der Hinterbühne garantiert zugleich die intersubjektive Übertragung reduzierter Komplexität. Die ko-produktive Erarbeitung einer Verteidigerstrategie neutralisiert effektiv Quellen möglichen Protests: Bevor der Laie in der mündlichen Verhandlung einem Konsistenzzwang durch die Ausübung seiner Verfahrensrolle ausgesetzt ist, bindet er sich bereits in Form einer rationalen Entscheidung an eine spezifische Form der Übernahme.

Der Angeklagte wird, sobald er sich selbst auf die Übernahme dieser Verhaltensprämisse gegen einen Strafnachlass festgelegt hat, hinsichtlich dieser geskripteten Form der Teilnahme an der mündlichen Verhandlung dauerhaft durch seinen Verteidiger kontrolliert. Die Inklusion in die Verfahrensrolle ist damit keine einmalige Änderung von Erwartungsstrukturen, sondern ein dauerhafter Prozess dieser professionellen Arbeit an der personalen Umwelt des Strafverfahrens bis zu dessen Abschluss.

Motiviert zur Übernahme der Verfahrensrolle wird der Angeklagte nicht durch die Ungewissheit des Ausgangs des Verfahrens, sondern durch das außerjuristische Motiv der Vermeidung einer drohenden hohen Strafe bei Non-Compliance. Äußerer Halt dieser Kommunikation liegt in der Konzeptualisierung der Justiz, 
insbesondere der Staatsanwaltschaft, als überlastet: Allgemeine Anerkennung innerhalb der Profession erfährt, dass in der Justiz eine Güterabwägung stattfindet zwischen effizienter Fallbearbeitung unter stark begrenzten personellen Ressourcen und rechtsstaatlichen Prinzipien. In der Folge werden - so die geteilte Annahme - Anklagen typischerweise nur dann erhoben, wenn die vorgeworfene Tat auch beweisbar ist. Protest innerhalb der Experten-Laien-Beziehung wird durch die Referenz auf die Deutungshoheit der „Justiz“ absorbiert. Die Ungewissheit des Ausgangs kann weder für den Mandanten noch für den Verteidiger Ausgangspunkt einer rationalen Verteidigungsstrategie sein. Unabhängig davon, ob ein jeweiliger Verteidiger diese Prämisse in ihrer Gültigkeit bei der Vermittlung bezweifelt oder nicht, muss im typischen Fall die Frage nach Schuld oder Unschuld vor dem Hintergrund notwendiger taktischer Überlegungen suspendiert werden:

\footnotetext{
„Aber einfach nur die Tatsache, dass falsche Geständnisse, auch hier in Deutschland, abgegeben werden, ja, auch ohne Folter und Druck, sondern einfach durch eine - naja Druck, Druck vielleicht schon - aber halt ohne Folter oder Drohung in dem Sinne - sondern einfach auch aus einer taktischen Überlegung. Und das ist natürlich ganz schlimm. Klar, wenn das rechtsstaatliche Verfahren auf der einen Seite gequetscht wird, dann kommt halt die Wurst auf der anderen Seite raus. Und das ist halt ein Problem. [W]enn halt auf der einen Seite klar ist, mein Mandant wird gegrillt, dann muss ich mir auf der anderen Seite überlegen, wie kann ich das möglichst verhindern.“ (RA11 66)
}

Diese informelle Akzeptanzbeschaffung auf Basis außerjuristischer Motive gibt, wie einleitend bemerkt, Außenstehenden wiederholt Anlass zu Kritik. Der DeLegitimation der Verfahrenspraxis wirkt entgegen, dass es zumindest in der mündlichen Verhandlung öffentlich zu einer freiwilligen Affirmation des maßgeblich durch den Richter erstellten Skripts kommt. In diesem Aspekt unterscheidet sich informelle Kommunikation im deutschen Strafverfahren auch signifikant vom US-amerikanischen System des „plea bargaining“. Im Fall des deutschen Strafverfahrens muss ein Geständnis auch in das Skript der mündlichen Verhandlung nachvollziehbar eingepflegt werden. Zwar stehen auch wie im US-amerikanischen Fall Justiz und Verteidigung sich gegenüber, sobald es aber zu einer Anklage kommt, wird dem informellen Tausch von Strafnachlass gegen Geständnis durch die Notwendigkeit der öffentlichen Aufführung Grenzen gesetzt. ${ }^{8}$

8 Kommt es jedoch nicht zur Anklage, dann weist die Zusammenarbeit zwischen Verteidigung und Staatsanwaltschaft in der Tat strukturelle Ähnlichkeit zum „plea bargaining“ auf, ohne wie dieses formalisiert zu sein (Dervan 2012; Devers 2011). Die Öffentlichkeit wird systematisch ausgeschlossen und das Verfahrensergebnis informell ausgehandelt. Dieser „Handel mit Gerechtigkeit“ (Schumann 1977) ist aber durch die dargestellten „Vergleichsmaßstäbe“ der Staatsanwalt- 
Durch die öffentliche Aufführung können auf der anderen Seite die Mechanismen von Legitimation durch Verfahren greifen. Auch wenn die Ungewissheit des Verfahrensausgangs nicht mehr Motor des Geschehens in der mündlichen Verhandlung war, zwingt sie zur rituellen Aufführung der informellen Absprache und überführt diese damit in eine öffentliche Verfahrensgeschichte. Auf sie kann die im Falle des Protests, der angesichts des typischerweise gelösten Inklusionsproblems ohnehin unwahrscheinlich ist, verwiesen werden.

\title{
6.c. Institutionalisierung informeller Kommunikation durch Mechanismen sozialer Schließung
}

Im System der Profession ist informelle Kommunikation mit Mechanismen sozialer Schließung geschützt: Professionsmitglieder im Strafverfahren werden innerhalb der Dyaden Staatsanwaltschaft-Verteidigung und Richterschaft-Verteidigung unterschieden in solche, mit denen informelle Kommunikation möglich ist, d.h. mit denen man „halt absolut offen reden kann“ (S7 79) und solche, die die dargestellten intersubjektiven Standards informeller Zusammenarbeit nicht teilen. Im Falle von Widerstand wird der Kontakt zum nicht-kooperierenden Professionsmitglied auf das formal Notwendige beschränkt. Insbesondere für Verteidiger ist dieses Bewertungsregime mit umfangreichen sozialen Folgen verknüpft, da diese dann unter hoher Unsicherheit eine Verteidigungsstrategie entwickeln müssen.

Die Staatsanwaltschaft entwickelt bei Nichtbefolgen der „konsensualen Linie“ (RA15 45) den Fall entlang interner Vergleichsmaßstäbe weiter und legt die Anklage dem Richter zur Weiterbearbeitung vor. Die Richterschaft bewältigt die durch Widerstand entstandene zusätzliche Komplexität durch eine strenge Einhaltung der StPO, nutzt also den ineffizienten Trichter des Verfahrens:

\begin{abstract}
„[A]lso, Rechtsanwälte, das ist in der Regel ein kollegiales Verhältnis und man arbeitet mit ihnen zusammen. Es gibt aber auch andere, die sogenannten Konfliktverteidiger. Die von vornherein, aus welchen Gründen auch immer, entweder, weil sie ihren Mandanten beeindrucken wollen oder weil sie das Verfahren verschleppen wollen, die versuchen, das Verfahren zu verzögern. Manchmal auch nur versuchen, die Rechte ihres Mandanten wahrzunehmen. Das nehmen wir dann aber manchmal auch als Konfliktverteidigung wahr. Da ist es dann ein eher ein distanziertes Verhältnis, was dann sich rein nach den Regeln der (lacht), auf der, dessen bewegt, was die StPO vorgibt.“ (R11 328)
\end{abstract}

schaft stark begrenzt, so können insbesondere „schwere Straftaten“ nicht zum Gegenstand eines solchen Vorgehens werden. 
Die Bezugnahme auf formale Verfahrensregeln, die im Falle einer „konstruktiven“ Zusammenarbeit nur habituell als Form - z. B. bei schriftlichen Urteilsbegründungen, Protokollen oder Belehrungen von Zeugen - Verwendung findet, erhält als Sanktionsinstrument eine neue Funktion, um „Lufthoheit“ (R10 177) zu bewahren. Von Seiten der Richtenden wird ein notwendig erhöhtes Zeitkontingent eingeplant.

Hält sich das Gericht streng an die formalen Regeln der StPO und kann es die zur Last gelegte Tat beweisen, erhält der Mandant das unter Verteidigern gefürchtete „Brett“ (RA12 58; RA16 93). Anschließender Protest lässt sich mit dem Verweis auf ein faires Verfahren an die gesellschaftliche Umwelt externalisieren. Zusätzlich wird von Seiten der Richterschaft angestrebt, durch die Verbreitung des Etiketts des „Konfliktverteidigers“ diesen von der Möglichkeit, sich kollegial mit Richtern eines Bezirks zu beraten, auszuschließen. Die Mechanismen sozialer Schließung greifen folglich nicht für jede verfahrensbeteiligte Rolle in der gleichen Intensität. Die mit der Rolle des Richters bzw. des Staatsanwaltes verbundenen Entscheidungsspielräume können nicht nur zu Zwecken der Fallarbeit dienen, sondern auch als Sanktionsmittel Verwendung finden.

Insbesondere der Erfolg eines Verteidigers ist in der Folge systematisch mit der Pflege von Kontaktsystemen und Entsprechung geteilter informeller Standards in der Fallarbeit verbunden, die die Übertragung reduzierter Komplexität in der mündlichen Verhandlung zeitökonomisch effizient sicherstellen. Daher tendieren Verteidiger dazu, dem Laien eine affirmative Selbstfestlegung nahezulegen und im Falle einer Abweichung vom geforderten Skript sich von dieser in der mündlichen Verhandlung implizit zu distanzieren, um sich nicht vor den „Lügenkarren“ (RA12 24) des Mandanten spannen zu lassen und sich vor Kollegen zum „Kasper“ (RA14 10) zu machen.

Zur Verallgemeinerung dieser These können die Ergebnisse der Studie von Altenhain/Dietmeier/May (2013) dienen: So hielten 63,1\% der befragten Verteidiger, aber lediglich 50\% der befragten Richter und 48,6\% der befragten Staatsanwälte Verfahrensabsprachen für ein unverzichtbares Instrument ihrer Praxis (2013, 56f.). Dabei sehen Verteidiger und Staatsanwälte gleichermaßen Richter als die Hauptprofiteure an $(2013,53)$. Die Frage danach, ob die Akzeptanz dieser informellen Erwartungsstrukturen auch mit der Internalisierung dazugehöriger Normen einhergeht, lässt sich vor dem Hintergrund aufgezeigter Rationalisierungsstrategien tendenziell bejahen.

Als prozessbeteiligter Jurist den informellen Erwartungsstrukturen nicht zu entsprechen ist folglich mit einem dreifachen Risiko behaftet: Der Ausgang des Verfahrens bleibt ungewiss, es können soziale Kosten entstehen und zusätzlich wird die Fallarbeit zeitintensiver. Ein Bruch mit informellen Erwartungsstruk- 
turen ist damit ein unwahrscheinliches Ereignis; unabhängig davon, ob gute Gründe dafür vorliegen, z. B. wenn ein Verteidiger wie im obigen Fall von der Unschuld seines Mandanten überzeugt ist, aber aus taktischen Gründen doch zu einem Geständnis rät.

\section{Diskussion der Ergebnisse}

Professionelle Kommunikation auf der Hinterbühne des Strafverfahrens ist systematisch mit den in „Legitimation durch Verfahren“ geschilderten Mechanismen, die sich auf der Vorderbühne der mündlichen Verhandlung vollziehen, verbunden. Die nichtöffentliche Kommunikation der kollegialen Fallbearbeitung und der Experten-Laien-Beziehungen erhält den Betrieb der Vorderbühne (zeitökonomisch) effizient am Laufen. Auf den „Trichter“ des Verfahrens als Mechanismus zur Reduktion von Komplexität und die Selbstfestlegung des Laien bei der öffentlichen Ableistung ,unbezahlter zeremonieller Arbeit“ wird dabei nicht prinzipiell verzichtet. Jedoch ist die spezifische Form der Übernahme der Verfahrensrolle durch den Laien professionell geskriptet. In der Folge müssen nur zentrale und nicht sämtliche Beweise eingeführt und auf die Vernehmung vieler Zeugen kann verzichtet werden. Inklusionsprobleme in das System des Strafverfahrens sind auf diese Weise gelöst. Die „Formalitäts-Informalitäts-Spanne“ zeichnet sich folglich entgegen der Annahme von Scheffer/Hannken-Illjes/Kozin $(2010,162)$ im typischen Fall dadurch aus, dass Komplexität umfangreich kollegial reduziert und Compliance durch Arbeit an der personalen Umwelt gesichert wird. Dies ist der systematische Unterbau, der es ermöglicht, die Formalstruktur des Strafverfahrens und damit hier die Rationalität des Rechts auf Dauer zu stellen.

Besonderes Charakteristikum dieses Verhältnisses im Strafverfahren ist, dass formale Vorgaben nicht nur in der Krisensituation untypischer Fälle zur Reduktion von Komplexität an Bedeutung gewinnen, wenn beispielsweise im Falle einer schweren Straftat tatsächlich der Ausgang des Verfahrens ungewiss ist. Darüber hinaus werden diese auch instrumentalisiert, um das Verhältnis informeller Kommunikation zu formalisierten Sinngehalten selbst vor Protest zu schützen. Mechanismen sozialer Schließung und die dazugehörige Bildung von Innen- und Außengruppen ermöglichen diejenigen Professionsmitglieder von den feldspezifischen Gewinnen auszuschließen, die nicht „konstruktiv“ bzw. „konsensual“ an der Fallbearbeitung mitwirken. Dabei ist es unwesentlich, ob es sich dabei um tatsächlichen Widerstand handelt oder lediglich als solcher interpretiert wird. Diese Mechanismen befördern damit die Normalisierung 
dieser Verhältnisse und gegebenenfalls die anschließende Internalisierung in der Berufssozialisation der Professionsmitglieder. Dieses System ist informell institutionalisiert und reicht weit über konkrete „Kontaktsysteme“ hinaus, wenngleich durch Kontaktsysteme Vertrauen generiert wird, das ermöglicht, dass rechtliche Graubereiche auch tiefer betreten werden können. Daraus folgt, dass Luhmanns Einschätzung von „Kontaktsystemen“ (Luhmann 1983, 75ff.) zwar zuzustimmen ist, dass diese keinen eigenständigen Beitrag zur Legitimation von Entscheidungen leisten. Für sich genommen haben sie nicht das Potential, die Reduktion von Komplexität und Übertragung reduzierter Komplexität sicherzustellen. Im Kontext des Systems der Profession erhalten diese aber die Funktion, die Institutionalisierung der Formalitäts-Informalitäts-Spanne durch Moderation der Reproduktion informeller Kommunikation zu befördern, was gemessen an der Binnenrationalität der Profession - zeitökonomisch effiziente Fallarbeit ermöglicht.

Durch diese Output-Orientierung der Justiz, an welcher funktional die Verteidigung mitwirkt und in welchem das Konditionalprogramm des Rechts von den Professionsmitgliedern als Ressource begriffen und genutzt wird, entsteht aber eindrücklich das einleitend dargestellte Legitimationsdilemma. Durch diese „Umkehrung des Konversionsprozesses“ (Offe 1974, 336) wird zwar die effiziente Bewältigung von Umweltkomplexität sichergestellt, zugleich aber die Legitimität daraus resultierender Entscheidungen auf der Vorderbühne systematisch in Frage gestellt. Besondere Gefahr geht von dem möglichen Protest aus, dass der Laie sich aus rein strategischen Gesichtspunkten vor dem Hintergrund einer etablierten Realitätsdefinition für eine spezifische Verteidigungsstrategie entscheidet - unabhängig von seiner Schuld oder Unschuld. Informelle Normen der kollegialen Fallbearbeitung stehen hier im eklatanten Widerspruch zu den formal-proklamierten Normen, denen das Rechtssystem verpflichtet ist. Daher bedarf es spezifischer Rationalisierungstrategien.

Die manifeste Rechtfertigung liegt in der Konzeptualisierung, durch welche Ressourcenknappheit der Justiz in ein ursächliches Verhältnis zur Objektivität der professionellen, insbesondere staatsanwaltschaftlichen, Reduktion von Komplexität gesetzt wird: Weil personelle Ressourcen knapp sind, werden typisch nur solche Fälle verfolgt, die eindeutig beweisbar sind, der Angeklagte also zweifelsfrei schuldig ist. Durch die Methoden des rentablen Filterns, der Zufriedenheit und der konfrontativen Vermittlung erbringt die Profession unter den gegebenen Umständen die beste aller möglichen Respezifikationsleistung von zu allgemein geratenen Kriterien. Daraus folgt, dass die These der Ökonomisierung der Justiz (Röhl 2009; Schulze-Fielitz 2002; Schütz 2005; Wassermann 2002) insofern zu spezifizieren ist, dass die perzipierte Ressourcenknappheit systematisch mit der Struktur informell-professioneller Kommunikation verwo- 
ben ist, durch diese reproduziert und damit als Teil der sozialen Wirklichkeit reifiziert wird. ${ }^{9}$

Die latente und zentrale Rechtfertigungsstrategie besteht jedoch darin, dass es letztendlich dem Beschuldigten bzw. Angeklagten überlassen bleibt, wie er auf die professionelle Realitätsdefinition reagiert. Die Festlegung auf eine Verteidigungsstrategie ist damit ebenso eine Selbstfestlegung. Es hätte diesem faktisch freigestanden, innerhalb der professionellen Realitätsdefinition eine irrationale Verteidigungsstrategie oder einen „Konfliktverteidiger“ aus der Außengruppe der Profession zu wählen. Dann muss aber der von der Entscheidung Betroffene sich auch mit den Folgen der Reduktion von Komplexität durch den sich im Verfahren aufbauenden Konsistenzzwang abfinden. In einem solchen Fall wird in Sinne der StPO die Ungewissheit des Ausgangs ostentativ durch die Professionsmitglieder gepflegt.

Erfolgreiches „Lernen im sozialen System“ der Profession bedeutet, die Prämissen der informell-kollegial etablierten Realitätsdefinition in Form einer funktionalen Verteidigungsstrategie bei der Übernahme der Verfahrensrolle anzuerkennen. Ist dieser Lernprozess nicht erfolgreich, wird die daraus entstehende Komplexität wieder dem sozialen System des Verfahrens überantwortet.

\section{Literatur}

Abbott, Andrew Delano (1988): The System of Professions. An Essay on the Division of Expert Labor. Chicago: University of Chicago Press.

Altenhain, Karsten/Dietmeier, Frank/May, Markus (2013): Die Praxis der Absprachen in Strafverfahren. Baden-Baden: Nomos.

Altenhain, Karsten/Hagemeier, Ina/Haimerl, Michael/Stammen, Karl-Heinz (2007): Die Praxis der Absprachen in Wirtschaftsstrafverfahren. Baden-Baden: Nomos.

Baer, Susanne (2015): Rechtssoziologie. Eine Einführung in die interdisziplinäre Rechtsforschung. Baden-Baden: Nomos.

Bogner, Alexander (2005): Das Experteninterview. Theorie, Methode, Anwendung. Wiesbaden: VS.

Brand, Christian; Petermann, Stefan (2010): Der „Deal“ im Strafverfahren, das Negativattest und die Beweiskraft des Protokolls. Neue Juristische Wochenschrift 63, 5, 268-271.

Bundesverfassungsgericht (2013): Urteil des Zweiten Senats vom 19. März 2013. http://www. bverfg.de/e/rs20130319_2bvr262810.html (zuletzt aufgerufen am 20.02.2020).

Corbin, Juliet/Strauss, Anselm L. (1990): Grounded Theory Research. Procedures, Canons and Evaluative Criteria. Zeitschrift für Soziologie 19, 6, 418-427.

$9 \mathrm{Zu}$ Verhandlung und Vermittlung im Gerichtsverfahren siehe auch die Beiträge von Jost (2017), Müller (2017) und Heck (2017) in diesem Heft. 
Dervan, Lucian (2012): Bargained Justice. Plea-bargaining's innocence problem and the Brady safety-valve. Utah Law Review 1, 51-97.

Devers, Lindsey (2011): Plea and Charge Bargaining. Research Summary. Arlington.

Eidmann, Dorothee (1994): Schlichtung. Zur Logik außergerichtlicher Konfliktregelung. Zwei Fallstudien. Baden-Baden: Nomos.

Elias, Norbert (1989): Studien über die Deutschen. Frankfurt a.M.: Suhrkamp.

Europäische Kommission (2018): Standard-Eurobarometer 89. Die öffentliche Meinung in der Europäischen Union. Anlage.

Europäische Menschenrechtskonvention (EMRK) (2010): Konvention zum Schutze der Menschenrechte und Grundfreiheiten vom 04.11.1950, zuletzt geändert durch Protokoll Nr. 14 vom 13.5.2004 m.W.v. 1.6.2010.

Fischer, Thomas (2010): Ein Jahr Absprache-Regelung. Praktische Erfahrungen und gesetzlicher Ergänzungsbedarf. Zeitschrift für Rechtspolitik 43, 8, 249-251.

Flick, Uwe (2008): Triangulation. Eine Einführung. Wiesbaden: VS.

Freidson, Eliot (2001): Professionalism. The Third Logic. Cambridge: Polity Press.

Gephart, Werner (Hrsg.) (2012): Rechtsanalyse als Kulturforschung. Frankfurt a.M.: Vittorio Klostermann GmbH.

Gläser, Jochen/Laudel, Grit (2009): Experteninterviews und qualitative Inhaltsanalyse als Instrumente rekonstruierender Untersuchungen. Wiesbaden: VS.

Goffman, Erving (1990): The Presentation of Self in Everyday Live. Harmondsworth: Penguin.

Hannken-Illjes, Kati (2006): Mit Geschichten argumentieren. Argumentation und Narration im Strafverfahren. Zeitschrift für Rechtssoziologie 2, 211-223.

Heck, Justus (2017): Vermittlung im Zivilverfahren. Zu Funktionen und Folgen undifferenzierter Konfliktbearbeitung, in diesem Heft.

Hettinger, Michael (2011): Die Absprache im Strafverfahren als rechtsstaatliches Problem. Juristenzeitung 66, 6, 292-301.

Hoffmann, Ludger (1983): Kommunikation vor Gericht. Tübingen: Gunther Narr Verlag.

Hoffmann, Ludger (2010): Wissensgenerierung: der Fall der Strafverhandlung, in: Ulrich Dausendschön-Gay (Hrsg.), Wissen in (Inter-)Aktion. Verfahren der Wissensgenerierung in Unterschiedlichen Praxisfeldern. Berlin: de Gruyter, 249-277.

Holzinger, Markus (2015): Informalisierung des Rechtsstaates? Über das Nebeneinander formaler und informaler Regelsysteme. Zeitschrift für Theoretische Soziologie 2, 1, 5-31.

Jost, Fritz (2017): Strategien der Bewältigung des Wahrheitsproblems im (Zivil-)Prozess, in diesem Heft.

Klatetzki, Thomas (2012): Professionelle Organisation, in: Maja Apelt/Veronika Tacke (Hrsg.), Handbuch Organisationstypen. Wiesbaden: VS, 165-182.

Kranenpohl, Uwe (2010): Hinter dem Schleier des Beratungsgeheimnisses. Der Willensbildungs- und Entscheidungsprozess des Bundesverfassungsgerichts. Wiesbaden: VS.

Kurtz, Thomas (2011): Der Professionsansatz von Niklas Luhmann. Soziale Systeme 17, 1, 31-52.

Latour, Bruno (2010): The making of law. An ethnography of the Conseil d'Etat. Cambridge: Polity.

Lautmann, Rüdiger (1970): Rolle und Entscheidung des Richters. Jahrbuch für Rechtssoziologie und Rechtstheorie 1, 381-416.

Lautmann, Rüdiger (1972): Justiz. Die Stille Gewalt. Teilnehmende Beobachtung und entscheidungssoziologische Analyse. Frankfurt a.M.: Athenäum.

Legnaro, Aldo/Aengenheister, Astrid (1999): Die Aufführung von Strafrecht. Kleine Ethnographie gerichtlichen Verhandelns. Baden-Baden: Nomos. 
Luhmann, Niklas (1964): Funktionen und Folgen formaler Organisation. Berlin: Duncker \& Humblot.

Luhmann, Niklas (1983): Legitimation durch Verfahren. Frankfurt a.M.: Suhrkamp.

Luhmann, Niklas (1997): Die Gesellschaft der Gesellschaft. Frankfurt a.M.: Suhrkamp.

Machura, Stefan (1993): Niklas Luhmanns „Legitimation durch Verfahren“ im Spiegel der Kritik. Zeitschrift für Rechtssoziologie 1, 97-114.

Machura, Stefan (2017): Legitimation durch Verfahren - Was bleibt?, in diesem Heft.

Maiwald, Kai-Olaf (2008): Die Fallperspektive in der professionellen Praxis und ihrer reflexiven Selbstvergewisserung. Allgemeine Überlegungen und ein empirisches Beispiel aus der Familienmediation. Forum Qualitative Sozialforschung 9, 3. http://www.qualitativeresearch.net/index.php/fqs/article/view/321 (zuletzt aufgerufen am 20.02.2020).

Mayring, Philipp (2015): Qualitative Inhaltsanalyse. Grundlagen und Techniken. Weinheim: Beltz.

Morlok, Martin/Kölbel, Ralf (2001): Rechtspraxis und Habitus. Rechtstheorie 32, 289-304.

Morlok, Martin/Kölbel, Ralf/Launhardt, Agnes (2000): Recht als soziale Praxis. Eine soziologische Perspektive in der Methodenlehre. Rechtstheorie 31, 15-46.

Müller, Marion (2017): Keine Legitimation durch Familiengerichtsverfahren? Akzeptanzprobleme gerichtlicher Entscheidungen und Konstruktion geschlechtsdifferenter Elternschaft, in diesem Heft.

Müller-Mall, Sabine (2012): Performative Rechtserzeugung. Eine theoretische Annäherung. Weilerswist: Velbrück Wissenschaft.

Nahrwold, Florian (2014): Die Verständigung im Strafverfahren. Eine rechtsvergleichende Untersuchung zwischen Deutschland und der Schweiz - zugleich ein Beitrag zum deutschen Verständigungsgesetz und zum abgekürzten Verfahren der Schweiz. Baden-Baden: Nomos.

Oevermann, Ulrich (1997): Theoretische Skizze einer revidierten Theorie professionalisierten Handelns, in: Arno Combe/Werner Helsper (Hrsg.), Pädagogische Professionalität. Untersuchungen zum Typus pädagogischen Handelns. Frankfurt a.M.: Suhrkamp, 70-182.

Offe, Claus (1974): Rationalitätskriterien und Funktionsprobleme politisch-administrativen Handelns. Leviathan 2, 3, 333-345.

Parsons, Talcott (1947): Introduction, in: Alexander Henderson/Talcott Parsons (Hrsg.), Max Weber. The Theory of Social and Economic Organization. Glencoe: Free Press, 58-60.

Pfister, Wolfgang (2013): Deal und Fehlurteil. Forensische Psychiatrie, Psychologie, Kriminologie 7, 4, 250-257.

Przyborski, Aglaja/Wohlrab-Sahr, Monika (2014): Qualitative Sozialforschung. Ein Arbeitsbuch. München: Oldenbourg.

Rehbinder, Manfred (2014): Rechtssoziologie. Ein Studienbuch. München: Beck.

Reichertz, Jo (Hrsg.) (1984): Sozialwissenschaftliche Analysen jugendgerichtlicher Interaktion. Tübingen: Stauffenburg Verlag.

Rensen, Hartmut (2017): Wie funktioniert die Interpretation des Rechts in der Praxis?, in: Verena Frick/Oliver W. Lemcke/Roland Lhotta (Hrsg.), Politik und Recht. Umrisse eines politikwissenschaftlichen Forschungsfeldes. Baden-Baden: Nomos, 41-62.

Röhl, Klaus F. (2009): Ökonomisierung der Justiz und richterliche Unabhängigkeit, in: Sächsisches Staatsministerium der Justiz und für Europa (Hrsg.), Impulse für eine moderne und leistungsstarke Justiz. Dokumentation. Symposium Justizlehre Dresden 2009. Stuttgart: Richard Boorberg Verlag, 103-118. 
Sachweh, Patrick (2010): Deutungsmuster sozialer Ungleichheit. Wahrnehmung und Legitimation gesellschaftlicher Privilegierung und Benachteiligung. Frankfurt a.M.: Campus-Verlag.

Schatzman, Leonard/Strauss, Anselm L. (1973): Field research. Strategies for a natural sociology. Englewood Cliffs: Prentice-Hall.

Scheffer, Thomas (2010): Ethnographie mit System am Beispiel von englischen Strafverfahren, in: René John/Anna Henkel/Jana Rückert-John (Hrsg.), Die Methodologien des Systems. Wie kommt man zum Fall und wie dahinter? Wiesbaden: VS, 141-160.

Scheffer, Thomas/Hannken-Illjes, Kati/Kozin, Alexander (2010): Criminal Defence and Procedure. Comparative Ethnographies in the United Kingdom, Germany, and the United States. Basingstoke: Palgrave Macmillan.

Schreiber-Klein, Ines (2015): Schuld gegen Effizienz. Deal or no Deal? Juristische Arbeitsblätter $12,888$.

Schulze-Fielitz, Helmuth (Hrsg.) (2002): Justiz und Justizverwaltung zwischen Ökonomisierungsdruck und Unabhängigkeit. Berlin: Duncker \& Humblot.

Schumann, Karl F. (1977): Der Handel mit Gerechtigkeit. Funktionsprobleme der Strafjustiz und ihre Lösungen - am Beispiel des amerikanischen plea bargaining. Frankfurt a.M.: Suhrkamp.

Schütz, Alfred/Luckmann, Thomas (2003): Strukturen der Lebenswelt. Konstanz: UVK.

Schütz, Carsten (2005): Der ökonomisierte Richter. Gewaltenteilung und richterliche Unabhängigkeit als Grenzen neuer Steuerungsmodelle in den Gerichten. Berlin: Duncker \& Humblot.

Schütze, Fritz (1978): Strategische Interaktion im Verwaltungsgericht. Eine soziolinguistische Analyse zum Kommunikationsverlauf im Verfahren zur Anerkennung als Wehrdienstverweigerer, in: Wolfgang Hoffmann-Riem (Hrsg.), Interaktion vor Gericht. Baden-Baden: Nomos, 19-100.

Seibert, Thomas-Michael (2004): Gerichtsrede. Wirklichkeit und Möglichkeit im forensischen Diskurs. Berlin: Duncker \& Humblot.

Starystach, Sebastian (2018): Die soziale Praxis des Gerichtsverfahrens. Über die juristische Fallbearbeitung in Straf- und Zivilverfahren. Wiesbaden: Springer VS.

Stegmaier, Peter (2009): Wissen, was Recht ist. Richterliche Rechtspraxis aus wissenssoziologisch-ethnografischer Sicht. Wiesbaden: VS.

Stegmaier, Peter (2013): Recht und Normativität aus soziologischer Perspektive, in: Julian Krüper (Hrsg.), Grundlagen des Rechts. Baden-Baden: Nomos, 65-87.

Strafprozessordnung (2016): Strafprozessordnung in der Fassung der Bekanntmachung vom 7. April 1987 (BGBI. I S. 1074, 1319), die zuletzt durch Artikel 2 des Gesetzes vom 21. November 2016 (BGBI. I S. 2615) geändert worden ist.

Theile, Hans (2012): Wahrheit, Konsens und § STPO § 257c StPO. Neue Zeitschrift für Strafrecht $32,12,666-671$.

Wassermann, Rudolf (2002): Ökonomismus versus Justizkultur. Justiz unter Ökonomisierungsdruck. Recht und Politik 38, 2, 241-245.

Wernet, Andreas (1997): Professioneller Habitus im Recht. Untersuchungen zur Professionalisierungsbedürftigkeit der Strafrechtspflege und zum Professionshabitus von Strafverteidigern. Berlin: Sigma.

Witzel, Andreas (2000): Das problemzentrierte Interview. Forum Qualitative Sozialforschung 1, $1,22$.

Wrase, Michael (2016): Zwischen Norm und sozialer Wirklichkeit. Zur Methode und Dogmatik der Konkretisierung materieller Grundrechtsgehalte. Berlin: Duncker \& Humblot. 
Wrase, Michael (2017 a): Recht als soziale Praxis. Eine Herausforderung für die juristische Profession?!, in: Arne Pilniok/Judith Brockmann (Hrsg.), Die juristische Profession und das Jurastudium. Baden-Baden: Nomos, 41-58.

Wrase, Michael (2017 b): Rechtsinterpretation als soziale Praxis. Eine rechtssoziologische Perspektive auf juristische Methodik, in: Verena Frick/Oliver W. Lemcke/Roland Lhotta (Hrsg.), Politik und Recht. Umrisse eines politikwissenschaftlichen Forschungsfeldes. Baden-Baden: Nomos, 63-84.

\section{Autoreninformation:}

\section{Dr. Sebastian Starystach}

Ruprecht-Karls-Universität Heidelberg, Max-Weber-Institut für Soziologie, Bergheimer Straße 58, 69115 Heidelberg, Deutschland, email: sebastian.starystach@soziologie.uni-heidelberg.de

Sebastian Starystach studierte Soziologie, Politikwissenschaft und Philosophie an den Universitäten Heidelberg und Wrocław. Seit seinem Abschluss im Jahr 2012 ist er wissenschaftlicher Mitarbeiter am Max-Weber-Institut für Soziologie der Universität Heidelberg und war von 2014 bis 2016 Gastdozent am Collège Universitaire de Sciences Po Campus in Nancy. 2017 promovierte er zum Thema „Die soziale Praxis des Gerichtsverfahrens“. Seine Forschungsschwerpunkte liegen neben der Rechtssoziologie in der Organisationssoziologie, Medizin- und Gesundheitssoziologie. Derzeit ist er Postdoktorand am Max-Weber-Institut für Soziologie im Projekt „Der Kampf gegen Korruption und Manipulation“. Neue Publikationen: Die soziale Praxis des Gerichtsverfahrens. Über die juristische Fallbearbeitung in Straf- und Zivilverfahren. Wiesbaden: VS, 2018; Organisationale Hindernisse auf dem Weg zur Profession. Eine wissenssoziologische Untersuchung über das berufliche Selbstverständnis von Pflegekräften im Krankenhaus. ARBEIT 27 (2018), 3, 191-212 (zusammen mit Stefan Bär/Carolin Ernst/Christina Streib); Feindliche Übernahme? Krankenhauspflege in Zeiten der Ökonomisierung. Kölner Zeitschrift für Soziologie und Sozialpsychologie 71 (2019), 2, 211-235 (zusammen mit Stefan Bär). 